\title{
Detection of Microcalcifications in Mammograms Using Nonlinear Subband Decomposition and Outlier Labeling
}

\author{
M. Nafi Gürcan, Yasemin Yardımcı, A. Enis Çetin \\ Bilkent University, \\ Dept. of Electrical and Electronics Engineering, \\ Bilkent, Ankara TR-06533, Turkey \\ E-mail: cetin@ee.bilkent.edu.tr \\ Rashid Ansari \\ Univ. of Illinois at Chicago \\ Department of Electrical Engineering \\ Chicago, Illinois, 60607-7053
}

\begin{abstract}
Computer-aided diagnosis (CAD) will be an important feature of the next generation Picture Archiving and Communication Systems (PACS). In this paper, computer-aided detection of microcalcifications in mammograms using a nonlinear subband decomposition and outlier labeling is examined. The mammogram image is first decomposed into subimages using a nonlinear subband decomposition filter bank. A suitably identified subimage is divided into overlapping square regions in which skewness and kurtosis as measures of the asymmetry and impulsiveness of the distribution are estimated. A region with high positive skewness and kurtosis is marked as a region of interest. Finally, an outlier labeling method is used to find the locations of microcalcifications in these regions. Simulation studies are presented.
\end{abstract}




\section{Introduction}

Computer-aided diagnosis (CAD) will be an important feature of the next generation Picture Archiving and Communication Systems (PACS). In this paper, computer-aided detection of microcalcifications in mammograms is considered. Presence of microcalcification clusters is an early sign of breast cancer in women though they can be easily overlooked by an examining radiologist due to their small sizes.

Recently a variety of schemes based on the wavelet transform for the computerized detection of microcalcifications have been proposed. ${ }^{1-3}$ In these schemes, the mammogram image is first passed through a subband decomposing filter bank. The subband images are weighted to enhance the microcalcification locations. A new image is reconstructed from the weighted sub-images. In the detection step, global and local gray-level thresholds are applied to the reconstructed image to extract possible microcalcification locations. These locations are grouped to identify microcalcification clusters. $\mathrm{In}^{1-3}$ the reconstructed image corresponds essentially to a bandpass filtered version of the original mammogram image and it should be noted that the detection techniques proposed in ${ }^{1-3}$ are based on subband decomposition using linear filter banks.

In our method microcalcification detection is carried out using a signal decomposition based on nonlinear filters. The method takes advantage of the recently proposed methods of nonlinear filter banks for application in compression. ${ }^{6-9}$ The study was motivated by the impulsive nature of the data characterizing the microcalcifications which are isolated well by nonlinear filters. The processing is simple, and does not require a full decomposition and reconstruction. Microcalcifications vary in size from $0.01 \mathrm{~mm}^{2}$ to $1.0 \mathrm{~mm}^{2}$ and they appear as short pulses. Since microcalcifications are small and isolated regions, they produce outliers in the high-band subsignal. The problem is then reduced to that of detecting outliers in the high-band component, obviating the need for signal reconstruction. Statistical procedures for detection of the microcalcifications are applicable at this stage. The highband subsignal is first divided into overlapping square regions in which skewness and kurtosis as measures of the asymmetry and impulsiveness of the distribution are estimated. A region with high positive skewness and kurtosis is marked as a region of interest. Finally, an outlier labeling method ${ }^{5}$ is used to find the locations of microcalcifications in these regions. The block diagram of the detection scheme is shown in Figure 1.

In Section 2, various nonlinear subband decomposition methods are briefly reviewed. The statistical detection procedure used in our method is described in Section 3. Simulation studies are presented in Section 4. Finally, conclusions are presented in Section 5. 


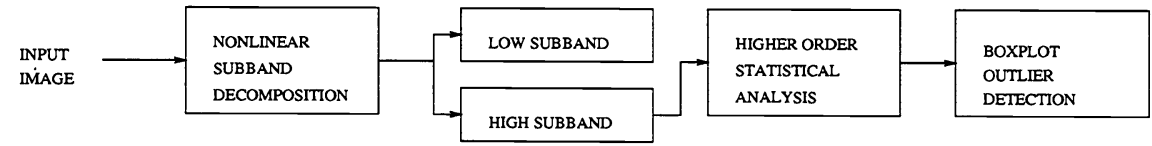

Figure 1: Microcalcification Detection Scheme

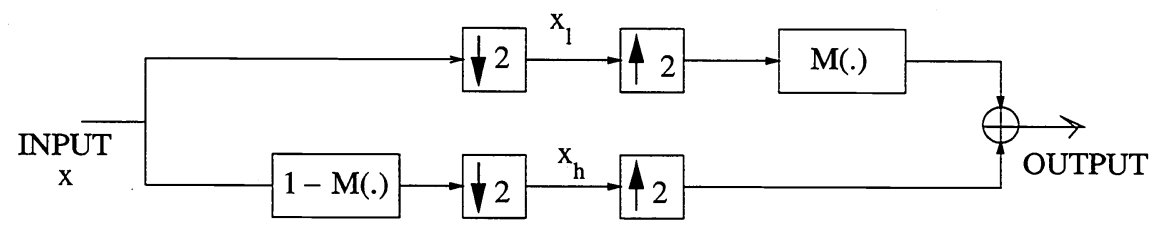

Figure 2: Nonlinear Subband Decomposition Structure in [6]

\section{Nonlinear Subband Decomposition Structures}

The subband decomposition using linear filters have been widely used in signal analysis and coding. Recently, the subband decomposition using nonlinear filters have been proposed and used in image coding. ${ }^{6-9}$ In this paper, the use of nonlinear subband decomposition in the analysis of mammogram images is investigated.

A nonlinear subband decomposition structure with perfect reconstruction property is shown in Figure 2. ${ }^{6}$ In the decomposition part, the input signal $x$ is just downsampled in the upper branch, while it is filtered by the nonlinear filter $I-M(\cdot)$ and then downsampled in the lower branch. The outputs after the downsampling by two are $x_{l}$ and $x_{h}$ which represent the low- and high-band sub-signals, respectively. The class of nonlinear filters providing perfect reconstruction is described in Reference. ${ }^{6}$

Another nonlinear subband decomposition structure with perfect reconstruction property is shown in Figure $3 .^{9}$ In this structure $H$ and $G$ are nonlinear operators, and they produce the low subband signal, $y_{a}$, and the detail high subband signal, $y_{d}$ from the input signal $\mathrm{x}(\mathrm{n})^{9}$ :

$$
\begin{aligned}
& y_{d}(n)=x_{2}(n)+H\left(\mathbf{x}_{1}(n)\right) \\
& y_{a}(n)=x_{1}(n)-G\left(\mathbf{y}_{d}(n)\right)
\end{aligned}
$$

where $x_{1}(n)=x(2 n-1), x_{2}(n)=x(2 n)$ and

$$
\begin{aligned}
& \mathbf{x}_{1}(n)=\left(x_{1}\left(n-N_{1}\right), \ldots, x_{1}(n), \ldots, x_{1}\left(n+N_{2}\right)\right) \\
& \mathbf{y}_{d}(n)=\left(y_{d}\left(n-N_{3}\right), \ldots, y_{d}(n), \ldots, y_{d}\left(n+N_{4}\right)\right),
\end{aligned}
$$

and $N_{1}, N_{2}, N_{3}, N_{4}$ are positive integers. This structure can be extended to two dimensions using either rectangular or quincunx subsampling methods. ${ }^{11,12}$

In this work $H$ is chosen to be a median filter with an $M \times M$ square region of support and $G$ is a closing filter ${ }^{10}$ with a $L \times L$ square structuring element. The support regions of the filters 


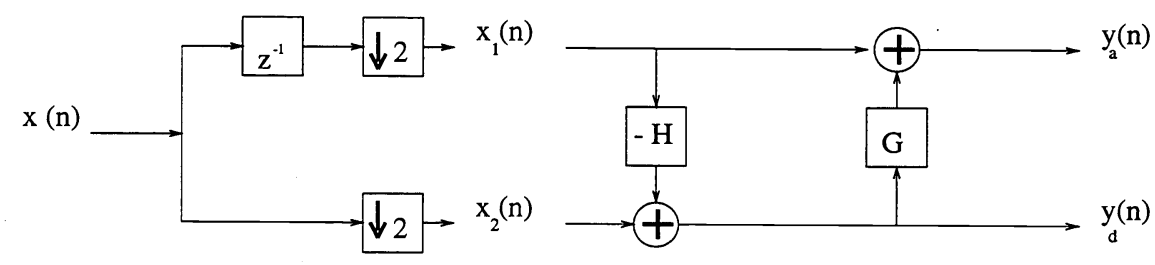

Figure 3: Nonlinear Subband Decomposition Structure in [9]

are chosen in accordance with typical dimensions of the microcalcifications. It should be noted that erosion and lower- $\alpha$ trimmed mean type filters can also be used for the $H$ filter. After the subband decomposition, the sub-signal $y_{a}$ will contain an enhanced version of the original signal while mainly microcalcifications are observed in the so-called highband sub-signal $y_{d}$.

\section{Statistical Detection Method}

An outlier is "an observation (or subset of observations) which appears to be inconsistent with the remainder of that set of data." ${ }^{4}$ Generally, due to the random nature of data, identifying and handling outliers is not an easy task. Nevertheless, there are numerous techniques available to detect and handle outliers. ${ }^{5}$ Since microcalcifications are tiny isolated regions, they produce outliers in the high-band subsignal. Therefore the microcalcification detection problem is equivalent to outlier detection in the high-band subimage.

In this paper, the detection is carried out in two steps. First, the highband subimage is divided into overlapping square regions. In these regions, skewness and kurtosis, measures of the asymmetry and impulsiveness of the distribution are estimated. If a region has high positive skewness and kurtosis then it is marked as a region of interest. In the second stage an outlier labeling method is used to find the locations of microcalcifications in these regions.

The computational complexity of the overall system is low. Rather than searching the whole image for outliers only regions with high susceptability are processed by the outlier labeling method.

\subsection{Skewness and Kurtosis Based Tests}

Skewness and kurtosis are higher order statistical parameters. ${ }^{13}$ For a random variable $\mathbf{x}$, the skewness is defined $\mathrm{as}^{14}$

$$
\gamma_{3}=\frac{E\left[(\mathbf{x}-E[\mathbf{x}])^{3}\right]}{\left(E\left[(\mathbf{x}-E[\mathbf{x}])^{2}\right]\right)^{3 / 2}}
$$


by:

and is a measure of the symmetry of the distribution. An estimate of the skewness is given

$$
\hat{\gamma_{3}}=\frac{\sum_{i=1}^{N}\left(x_{i}-\hat{m}\right)^{3}}{(N-1) \hat{\sigma}^{3}}
$$

where $\hat{m}$ and $\hat{\sigma}$ are the estimates of the mean and standard deviation over $N$ observations $x_{i}$ $(i=1, \ldots, N)$.

Similarly, for a random variable $\mathbf{x}$ the kurtosis is defined as

$$
\gamma_{4}=\frac{E\left[(x-E[x])^{4}\right]}{\left(E\left[(x-E[x])^{2}\right]\right)^{2}}-3
$$

and is a measure of the heaviness of the tails in a distribution. An estimate of the kurtosis is given by:

$$
\hat{\gamma}_{4}=\frac{\sum_{i=1}^{N}\left(x_{i}-\hat{m}\right)^{4}}{(N-1) \hat{\sigma}^{4}}-3
$$

where $\hat{m}$ and $\hat{\sigma}$ are defined as before. For the Gaussian distribution $\gamma_{3}$ and $\gamma_{4}$ are equal to zero.

If a region contains microcalcifications then due to the impulsive nature of microcalcifications the symmetry of the distribution of highband subimage coefficients is destroyed as shown in Figure 4. It is also evident that the tails of the distribution are heavier and hence the kurtosis assumes a high value. Therefore a statistical test based on skewness and kurtosis is effective in finding regions with asymetrical and heavier tailed distributions. The detection problem is posed as an hypothesis testing problem in which the null hypothesis, $H_{o}$, corresponds to the case of no microcalcifications against the alternative $H_{1}$ :

- $H_{o}: \gamma_{3}<T_{1}$ or $\gamma_{4}<T_{2}$

- $H_{1}: \gamma_{3} \geq T_{1}$ and $\gamma_{4} \geq T_{2}$

where $T_{1}$ and $T_{2}$ are experimentally determined thresholds.

Once the regions containing microcalcifications are determined by the above test the locations of the microcalcifications are estimated by the outlier labeling method described in the next subsection.

\subsection{Boxplot Outlier Labeling Method}

In this work, we used the so-called boxplot outlier labeling method ${ }^{5}$ which is available in most of the statistical software packages. In this method data $\mathbf{x}$ is first rank ordered, 

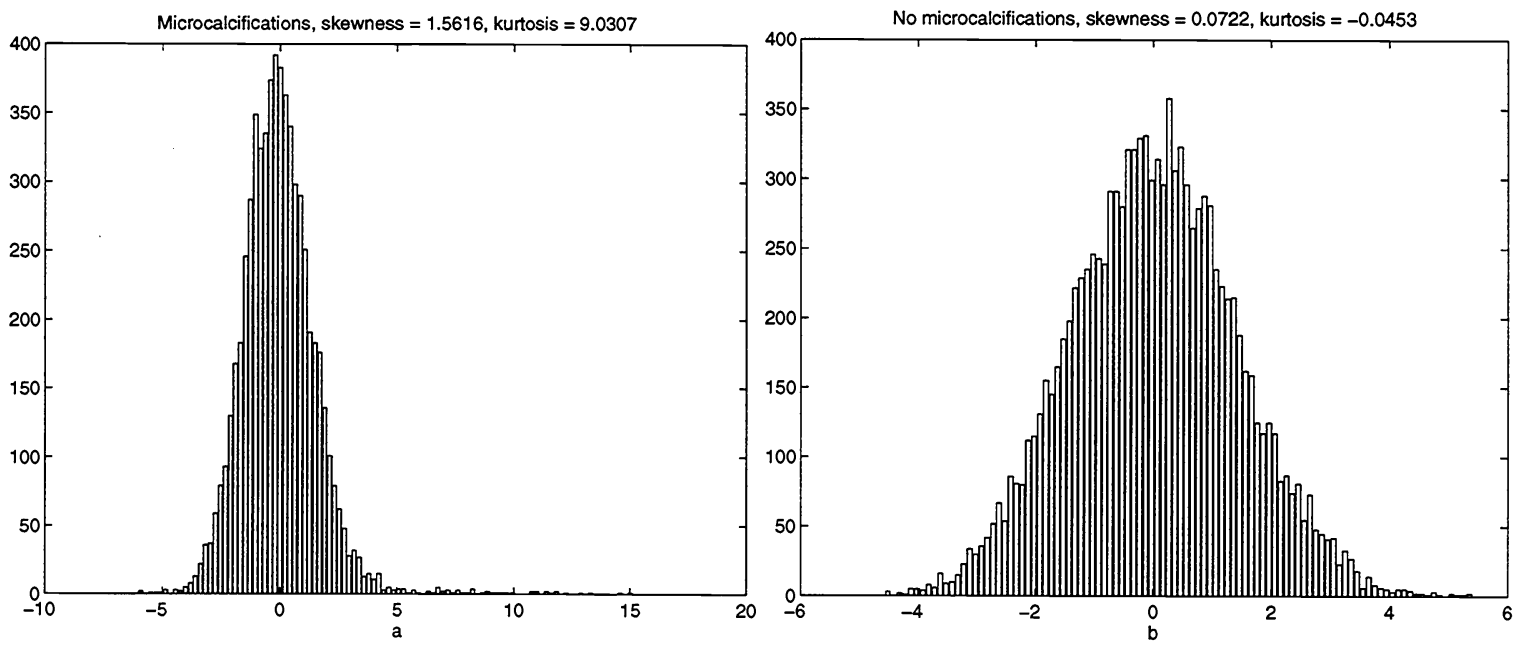

Figure 4: Sample value distributions in regions with (a) microcalcifications and (b) no microcalcification in the bandpass subband image

$\mathbf{x}=\left\{x_{1}, x_{2}, \ldots ., x_{n}\right\}$. Next, the median, the lower quartile, $Q_{1}$ and the upper quartile $Q_{3}$ values are determined through the following formulas in Equations 7-9.

$$
\begin{aligned}
f & =\frac{\lfloor(n+1) / 2\rfloor+1}{2} \\
Q_{1} & =x_{(f)} \\
Q_{3} & =x_{(n+1-f)}
\end{aligned}
$$

where $\lfloor y\rfloor$ represents the greatest integer less than or equal to $y$. The interquartile range $R_{F}$ is defined to be $Q_{3}-Q_{1}$. The boxplot method determines the outliers to be the part of data which is outside the region $\left(Q_{1}-k R_{F}, Q_{3}+k R_{F}\right)$. The parameter $k$ is usually taken to be 1.5 or 3.0. Figure 5 illustrates the boxplot outlier labeling method.

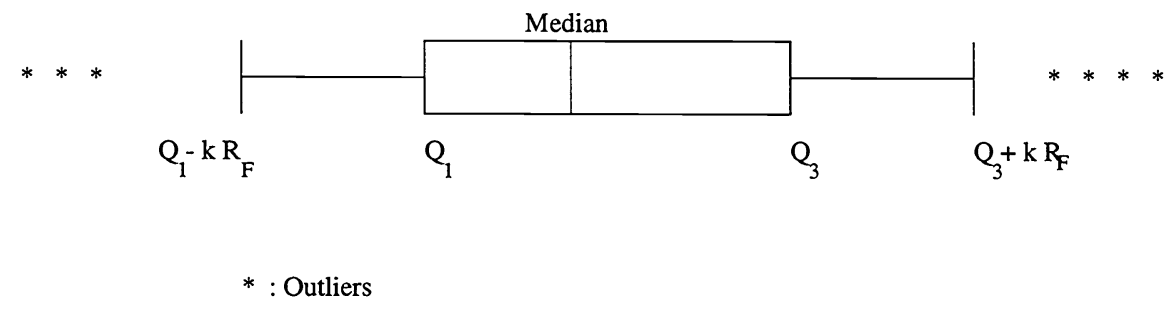

Figure 5: Boxplot outlier labeling method definitions 


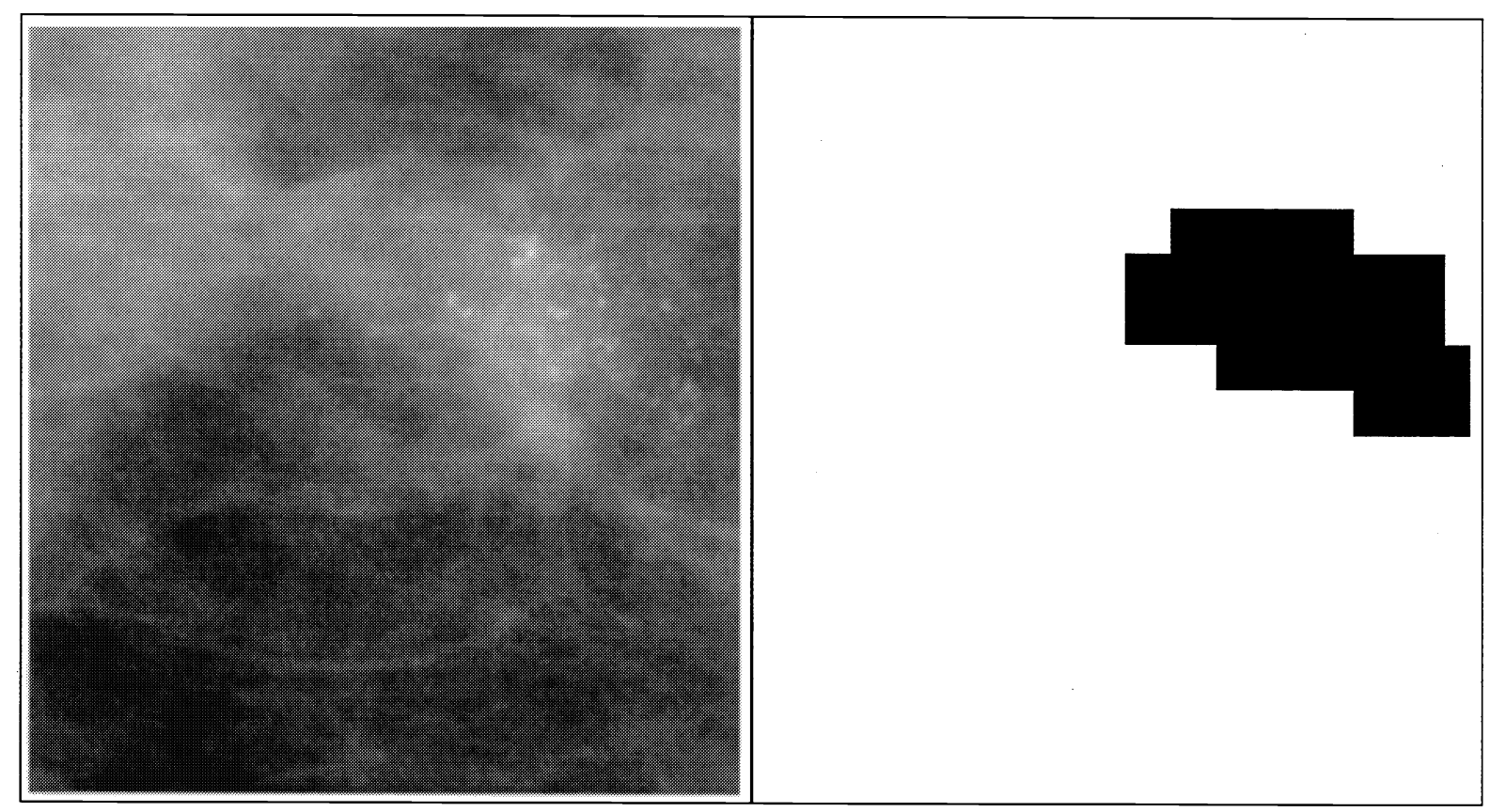

Figure 6: (a) A part of a mammogram image, (b) Regions with microcalcificati ons

\section{Simulation Results}

In this paper, simulation studies are carried out on mammogram images taken from a set digitized by Nico Karssemeijer of University Hospital Nijmegen, The Netherlands. Each image in the set also contains a ground truth file, in which the regions with microcalcifications are indicated by expert radiologists. In Figure 6(a) a part of a mammogram image is shown. This particular image contains a cluster of microcalcifications. The image is first processed by the nonlinear filter bank and the highband subimage is produced. The support of the median filter is a $21 \times 21$ region. In Figure 6(b), the result of the higher order statistical hypothesis testing algorithm is shown. The black squares indicate suspicious regions. The proposed detection scheme is successful in finding all the critical regions in this example and in tests with 10 different mammogram images of size $2048 \times 2048$. The size of the square regions is chosen to be $30 \times 30$ with an overlap of 15 . The experimentally determined thresholds for skewness $\hat{\gamma}_{3}$ and kurtosis $\hat{\gamma}_{4}$ are 1.0 and 2.0 , respectively.

Boxplot outlier labeling method is applied to the suspicious regions determined by the hypothesis testing. For example, Figure 7 shows a horizontal line of mammogram image which is known to contain a microcalcification. In the same figure the middle plot depicts the difference between the original signal and its median filtered version. This difference plot corresponds to the high-subband of the nonlinear decomposition structure. The bottom plot illustrates the output of the outlier detection scheme. Figure 8 shows the output of the detection scheme on a part of the mammogram image with a microcalcification cluster. In both cases the microcalcifications 

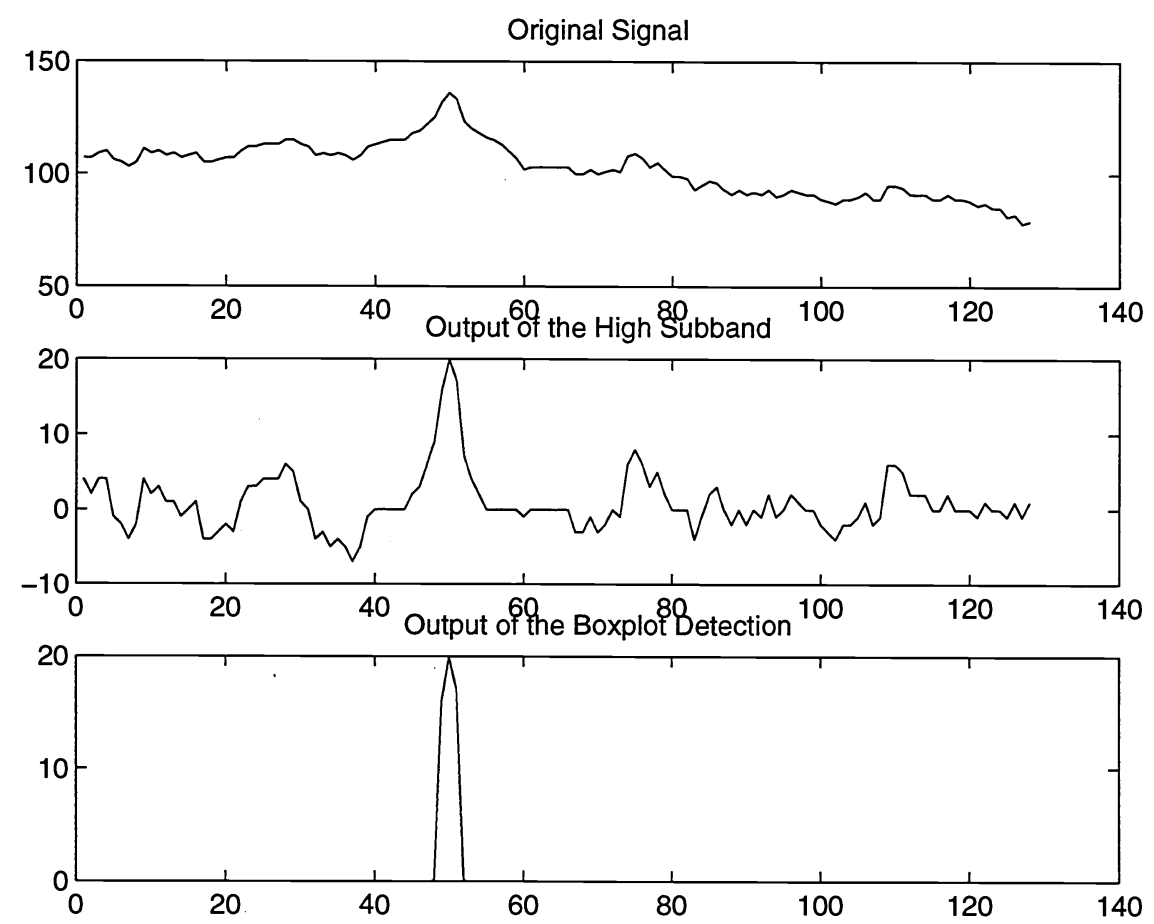

Figure 7: (a) A horizontal line of the mammogram image which is known to contain a microcalcification, (b). high-band sub-signal $x_{h}$, (c) output of the outlier detection method.

are successfully detected.

\section{Conclusions}

Computer-aided diagnosis (CAD) will be an important feature of the next generation Picture Archiving and Communication Systems (PACS). In this paper, automatic detection of microcalcifications in mammogram images is considered. The mammogram image is first processed by a nonlinear subband decomposition filter bank. Microcalcifications, tiny, isolated regions, produce outliers in the highband subimage. Next, the so-called highband subimages is divided into overlapping square regions in which skewness and kurtosis are estimated. The higher order statistical parameters, skewness and kurtosis are measures of the asymmetry and impulsiveness of the distribution. Therefore a region with high positive skewness and kurtosis is marked as a region of interest. Finally, an outlier labeling method is used to find the locations of microcalcifications in these regions.

Simulation results show that this method is successful in detecting regions with microcalcifications. 


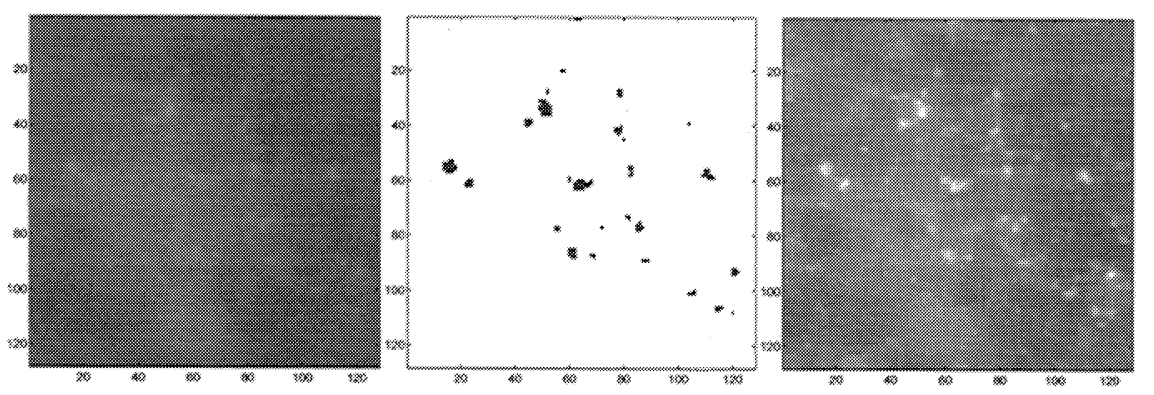

Figure 8: (a) A region of a mammogram image containing microcalcifications, (b) output of the detection scheme (c) enhanced image $y_{a}$ (the low subband image) in Figure 3.

\section{REFERENCES}

[1] H. Yoshida, K. Doi, R. M. Nishikawa, "Automated detection of clustered microcalcifications in digital mammograms using wavelet transform techniques," Proc. SPIE Int. Conf. on Visual Commun. and Image Proc., 2167:868-886, 1994.

[2] H. Yoshida, W. Zhang, W. Cai, K. Doi, R. M. Nishikawa and M. L. Giger, "Optimizing Wavelet Transform Based On Supervised Learning For Detection of Microcalcifications in Digital Mammograms," Proceedings of IEEE International Conference on Image Processing, pp. 152-155 1995.

[3] R. N. Strickland, H. I. Hahn, "Wavelet Transform Matched Filters For the Detection and Classification of Microcalcifications in Mammography," Proceedings of IEEE International Conference on Image Processing, pp. 422-425, 1995.

[4] Y. Barnett, T. Lewis, Outliers in Statistical Data, 3rd Ed. New York: John Wiley \& Sons, 1994.

[5] B. Iglewicz, D. C. Hoaglin, How to Detect and Handle Outliers, ASQC basic references in quality control; v.16, 1993.

[6] O. Egger, M. Kunt, "Embedded Zerotree Based Lossless Image Coding," IEEE ICIP'95, vol. II pp. 616-619., June 1995.

[7] D. E. F. Florencio, R. Schafer "Perfect Reconstructing Nonlinear Filter Banks," in Proc. of IEEE Int. Conf. on Acoust., Speech, Signal Proc., 1996.

[8] R. L. de Queiroz, D. A. F. Florencio, "A Pyramidal Coding Using a Nonlinear Filter Bank," in Proc. of IEEE Int. Conf. on Acoust., Speech, Signal Proc., 1996.

[9] F. J. Hampson, J. C. Pesquet, "A Nonlinear Subband Decomposition with Perfect Reconstruction," in Proc. of IEEE Int. Conf. on Acoust., Speech, Signal Proc., 1996. 
[10] I. Pitas, A. N. Venetsanopoulos, Nonlinear Digital Filters - Principles and Applications, Massachusetts: Kluwer Academic Publishers, 1990.

[11] R. Ansari, A. E. Cetin, S. H. Lee, "Sub-band Coding of Images Using Nonrectangular Filter Banks," SPIE Vol. 974, pp. 315-322, Applications of Digital Image Processing XI, 1988.

[12] M. Phoong, C. W. Kim, P.P. Vaidyanathan and R. Ansari, "A new class of two-channel biorthogonal filter banks and wavelet bases," IEEE Trans. on Signal Proc., March 1995, pp. 649-665, vol. 43, No. 3 .

[13] C. L. Nikias, A. Petropulu ,Higher Order Statistical Analysis, Prentice Hall, 1994.

[14] P. J. Bickel, K. A. Doksum, Mathematical Statistics, Holden-Day California : 1977. 